\title{
Type-III and Tilted Dirac Cones Emerging from Flat Bands in Photonic Orbital Graphene
}

\author{
M. Milićević, ${ }^{1}$ G. Montambaux, ${ }^{2}$ T. Ozawa, ${ }^{3}$ O. Jamadi, ${ }^{4}$ B. Real,${ }^{4}$ I. Sagnes, ${ }^{1}$ A. Lemaitre, ${ }^{1}$ \\ L. Le Gratiet, ${ }^{1}$ A. Harouri, ${ }^{1}$ J. Bloch, ${ }^{1}$ and A. $\mathrm{Amo}^{4}$ \\ ${ }^{1}$ Centre de Nanosciences et de Nanotechnologies (C2N), CNRS Université Paris-Sud/Paris-Saclay, \\ Palaiseau, France \\ ${ }^{2}$ Laboratoire de Physique des Solides, CNRS, Université Paris-Sud, Université Paris-Saclay, \\ 91405 Orsay Cedex, France \\ ${ }^{3}$ Interdisciplinary Theoretical and Mathematical Sciences Program (iTHEMS), \\ RIKEN, Wako, Saitama 351-0198, Japan \\ ${ }^{4}$ Université de Lille, CNRS, UMR 8523-PhLAM-Physique des Lasers Atomes et Molécules, \\ F-59000 Lille, France
}

(Received 2 December 2018; revised manuscript received 16 May 2019; published 23 July 2019)

\begin{abstract}
The extraordinary electronic properties of Dirac materials, the two-dimensional partners of Weyl semimetals, arise from the linear crossings in their band structure. When the dispersion around the Dirac points is tilted, one can predict the emergence of intricate transport phenomena such as modified Klein tunneling, intrinsic anomalous Hall effects, and ferrimagnetism. However, Dirac materials are rare, particularly with tilted Dirac cones. Recently, artificial materials whose building blocks present orbital degrees of freedom have appeared as promising candidates for the engineering of exotic Dirac dispersions. Here we take advantage of the orbital structure of photonic resonators arranged in a honeycomb lattice to implement photonic lattices with semi-Dirac, tilted, and, most interestingly, type-III Dirac cones that combine flat and linear dispersions. Type-III Dirac cones emerge from the touching of a flat and a parabolic band when synthetic photonic strain is introduced in the lattice, and they possess a nontrivial topological charge. This photonic realization provides a recipe for the synthesis of orbital Dirac matter with unconventional transport properties and, in combination with polariton nonlinearities, opens the way to study Dirac superfluids in topological landscapes.
\end{abstract}

DOI: 10.1103/PhysRevX.9.031010

Subject Areas: Graphene, Photonics,

Topological Insulators

\section{INTRODUCTION}

The extraordinary transport properties of Dirac materials arise from the spinor nature of their electronic wave functions and from the linear dispersion around Dirac and Weyl points. In two dimensions, Klein tunneling, weak antilocalization, unconventional Landau levels, or bulk pseudoconfinement appear as some of their most remarkable features $[1,2]$. Standard Dirac cones, like those present in graphene and other two-dimensional materials, have rotational symmetry about the Dirac quasimomentum. Their topological properties make them particularly robust to deformations of the lattice: Dirac cones always appear in pairs, each of them characterized by a topological charge [3]; in the presence of time-reversal and inversion symmetry,

Published by the American Physical Society under the terms of the Creative Commons Attribution 4.0 International license. Further distribution of this work must maintain attribution to the author(s) and the published article's title, journal citation, and DOI. they can only be annihilated via their merging with a Dirac cone of opposite charge [4-9].

Dirac cones can be classified according to the geometry of their Fermi surface. The cylindrically symmetric cones described above belong to the family of type-I Dirac cones. They are characterized by a closed Fermi surface eventually becoming a single point at the band crossing, where the density of states vanishes [Fig. 1(a)]. However, they are not the only kind of linear band crossings that can be found in Dirac materials. The general Hamiltonian describing a Dirac cone in two dimensions can be expressed as [10]

$$
H(\mathbf{q})=\left(v_{0 x} q_{x}+v_{0 y} q_{y}\right) \sigma_{0}+v_{x} q_{x} \sigma_{a}+v_{y} q_{y} \sigma_{b},
$$

where $q_{x, y}$ is the wave vector measured from the Dirac point; $v_{0 x}, v_{0 y}, v_{x}$, and $v_{y}$ represent effective velocities; $\sigma_{0}$ is the $2 \times 2$ identity matrix; and $\sigma_{a, b}=\mathbf{u}_{a, b} \cdot \boldsymbol{\sigma}$, where $\mathbf{u}_{a, b}$ are suitably chosen orthogonal unit vectors and $\boldsymbol{\sigma}=\left(\sigma_{x}, \sigma_{y}, \sigma_{z}\right)$ is the vector of Pauli matrices. Throughout the entire paper, we take $\hbar=1$. The eigenenergies of this Hamiltonian form two bands: 


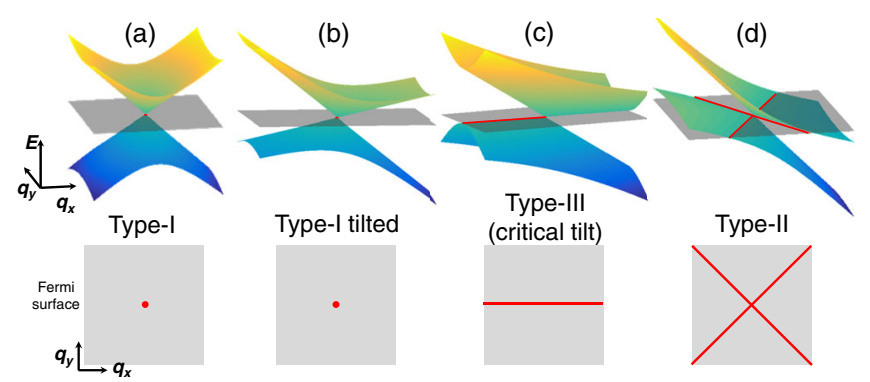

FIG. 1. Types of Dirac dispersions in two dimensions. (Top) Dispersions together with the zero-energy plane (grey). (Bottom, in red) Zero-energy Fermi surface. (a) Standard, type-I Dirac cones characterized by a linear dispersion in all directions in $k$ space and a pointlike Fermi surface. (b) Type-I tilted Dirac cone. (c) Type-III Dirac point (critically tilted), combining flat band and linear dispersions. Its Fermi surface is a line. (d) Type-II Dirac cone.

$$
E_{ \pm}(\mathbf{q})=v_{0 x} q_{x}+v_{0 y} q_{y} \pm \sqrt{\left(v_{x} q_{x}\right)^{2}+\left(v_{y} q_{y}\right)^{2}}
$$

If both coefficients $v_{0 x}$ and $v_{0 y}$ are equal to zero, we obtain the energy spectrum of a type-I Dirac cone with Fermi velocities $v_{x}$ and $v_{y}$. If any of the $v_{0 x}, v_{0 y}$ coefficients is nonzero, then the Dirac cone is tilted [Fig. 1(b)]. In twodimensional materials, this kind of tilted Dirac dispersion has been predicted to appear in quinoid-type [10] and hydrogenated graphene [11], and it has been indirectly evidenced in the organic semiconductor $[10,12,13]$ $\alpha-(\mathrm{BEDT}-\mathrm{TTF})_{2} I_{3}$. The interest of materials presenting this dispersion is its nonisotropic transport properties, which can be used for valley filtering in p-n junctions [14] or for the generation of a photocurrent [15].

When the tilt parameter $\tilde{v}_{0} \equiv \sqrt{\left(v_{0 x} / v_{x}\right)^{2}+\left(v_{0 y} / v_{y}\right)^{2}}$ is larger than 1, a type-II Dirac point [10,16,17] is formed [Fig. 1(d)]. Its Fermi surface is no longer a point but two crossing lines, and the density of states at the energy of the Dirac point becomes finite [16]. They have recently been observed in the form of Fermi arcs in three-dimensional semimetals [18-20]. A particularly interesting situation takes place at the transition between type-I and type-II Dirac cones, that is, when the tilt parameter $\tilde{v}_{0}=1$. In this case, the cone is critically tilted, with a flat band along one direction [Fig. 1(c)]. Because of its distinct Fermi surface, a single line, and its diverging density of states, this kind of dispersion has been labeled a type-III Dirac cone [21,22]. At the Fermi energy, the quasiparticles in these kinds of cones present highly anisotropic masses with localized states along one direction in the flat band coexisting with massless states moving ballistically. While most of their electric and magnetic properties are yet to be unveiled, they have been predicted to greatly enhance the superconducting gap in Weyl semimetals [23], and they provide a new platform for the study of correlated phases with a flat band [24]. Moreover, the possibility of implementing twodimensional heterostructures with different kinds of Dirac cones would open the door to the study of analogue black holes in a solid-state environment [21,25-27].

Type-III Dirac points have not yet been reported experimentally, and tilted type-I and type-II Dirac points have been challenging to synthesize $[13,18,20]$ because they require materials whose constituent atoms are arranged in lattices with intricate electron hoppings $[10,12,16]$. Existing proposals rely on the use of stratified media [28], or on the engineering of next-nearest-neighbor tunneling, which is difficult to find in natural materials and to implement in electronic metamaterials. Artificial photonic lattices represent an opportunity to explore the physics of unconventional Dirac points thanks to the at-will control of on-site energies and hoppings [29]. Current schemes are based on the design of long-distant coupling of photons in lattices of resonators [30,31] and the use of screw symmetries [32]. Using more sophisticated approaches, evidence of type-II Weyl points has recently been reported in microwave metamaterials $[33,34]$ and via conical diffraction in laser-written waveguides with elaborate couplings [35]. In most of these cases, the tilted Dirac cones appear as a consequence of band inversions [30,31], a situation in which the magnitude of the tilt cannot be easily controlled. This has been the main reason preventing the observation of type-III Dirac cones.

In this article, we propose and demonstrate experimentally a method to implement tilted and semi-Dirac cones for photons, and we provide the first experimental observation of type-III Dirac cones. We employ $p_{x}, p_{y}$ orbital bands in a honeycomb lattice of polariton micropillars, which arise from the nearest-neighbor hopping of photons confined in the first excited modes of each resonator of the lattice [36,37]. In this analogue system, the band structure is directly accessible in photoluminescence experiments. The orbital bands present a flat band that touches a parabolic band. When asymmetry in the hopping is introduced, which simulates uniaxial strain in solid-state graphene, the flat-parabolic band touching evolves into tilted and type-III Dirac cones. The fact that they emerge from a flat band makes type-III Dirac cones particularly robust to changes in the hopping, as we show below. The richness of this multiband system allows, in addition, the observation of semi-Dirac cones, which combine massless and massive dispersions. By analyzing their topological charge, i.e., the winding of the Hamiltonian around each Dirac point, we show that the semi-Dirac, tilted, and type-III Dirac cones emerge as a consequence of topological Lifshitz transitions induced by strain in the orbital bands. The present realization shows the potential of orbital bands to engineer the properties of Dirac photonic matter and may inspire new routes towards the synthesis of electronic Dirac materials. 


\section{PHOTONIC ORBITAL LATTICE}

The photonic platform we employ is a honeycomb lattice of coupled micropillars. The lattice is etched from a semiconductor planar microcavity made out of two AlGaAs Bragg mirrors that confine photons in the vertical direction and twelve GaAs quantum wells embedded in the spacer between the mirrors (see the Appendix A). At $10 \mathrm{~K}$, the temperature of our experiments, the confined photons, and the quantum-well excitons are in the strong coupling regime and form polaritons, light-matter hybrid quasiparticles. Each individual micropillar [Fig. 2(a)], with a diameter of $2.75 \mu \mathrm{m}$, presents an additional lateral confinement due to the index of refraction contrast between the semiconductor and air. Figure 2(b) shows the characteristic discrete photonic spectrum of a single micropillar, with the lowest-energy $s$ mode being cylindrically symmetric, and the first excited modes formed by two $p_{x}, p_{y}$ orbitals with lobes oriented $90^{\circ}$ from each other and a node at the center of the pillar [37]. Note that in Fig. 2(b), the $p$ modes show a small splitting arising from small anisotropy in the shape of the pillar and from polarization-momentum coupling characteristic of semiconductor microcavities [38,39].

In the honeycomb lattice, the micropillars overlap (center-to-center distance $d=2.4 \mu \mathrm{m}$ ) enabling the hopping of photons between adjacent sites [Fig. 2(d)]. The coupling of $s$ modes results in two bands with spectra and eigenmodes very similar to those of electrons in graphene, shown in the low-energy part of Fig. 2(e) and studied in previous works [36,40]. Here, we concentrate on the orbital bands that arise from the coupling of $p$ modes [high-energy set of bands in Fig. 2(e)]. Orbitals oriented along the link between adjacent pillars $\left[p_{x}\right.$ in the example of Fig. 2(c)] present a coupling $t_{L}$ much stronger than $t_{T}$, the coupling of orbitals oriented perpendicular to the link [ $p_{y}$ in Fig. 2(c)].
Figure 3(a) shows the angle-resolved photoluminescence of the $p$ bands when exciting the lattice at its center with a nonresonant continuous-wave laser at $745 \mathrm{~nm}$, focused on a $3-\mu \mathrm{m}$-diameter spot. The power of the laser is $6 \mathrm{~mW}$, well below the threshold for any nonlinear effect. The laser creates electrons and holes in the quantum wells that relax down through phonon scattering to form polaritons distributed over the different bands of the lattice. When polaritons recombine via the escape of a photon out of the microcavity, photons are emitted with an in-plane momentum and a frequency that correspond to those of the original polaritons within the lattice of resonators. An angle- and energy-resolved measurement using an imaging spectrometer coupled to a CCD allows one to reconstruct the dispersion relation [41]. We select the emission linearly polarized along the $k_{y}$ direction. Similar results were obtained for the orthogonal polarization. To avoid destructive interference effects along high-symmetric crystallographic directions, characteristic of bipartite lattices [42], we record the emission as a function of $k_{y}$ for $k_{x}=4 \pi / 3 d$, passing through the $K, \Gamma$, and $K^{\prime}$ points in the second Brillouin zone [dashed line in Fig. 3(e)]. Four bands are observed: The lowest one is flat, while the two central bands present two type-I Dirac points at the $K$ and $K$ ' points and touch the flat band at the $\Gamma$ point [36]. This is in good agreement with a nearest-neighbor tight-binding calculation [43] assuming $t_{T}=0$ (white solid lines, see Appendix B). The values of $t_{L}$ in the investigated lattices range from $-0.65 \mathrm{meV}$ to $-0.90 \mathrm{meV}$, resulting in $p$ bands with a total bandwidth between 2 and $3 \mathrm{meV}$. As the polarization-related splitting apparent in the $p$ modes of Fig. 2(b) is much smaller $(0.3 \mathrm{meV})$ than the bandwidth, we neglect its influence in the analysis of our results. Note that in the experiments, the uppermost band deviates from a flat band due to the coupling to higher-energy bands (arising from photonic $d$ orbitals in the micropillars).
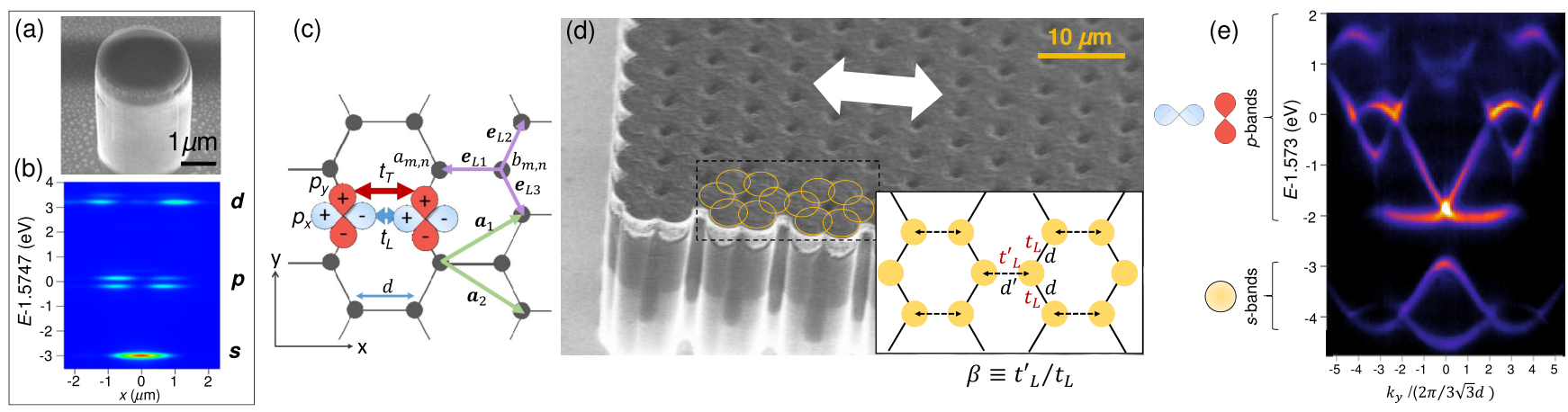

FIG. 2. Honeycomb polariton lattice with orbital bands. (a) Scanning electron microscopy image of a single micropillar, the elementary building block of the honeycomb lattice. (b) Characteristic emission spectrum of a single micropillar, showing $s, p$, and $d$ discrete modes. The micropillar has been etched from the same wafer as the lattices studied in this work. (c) Scheme of the coupling of $p_{x}$ and $p_{y}$ orbitals in the honeycomb lattice with hoppings $t_{L} \gg t_{T}$. (d) Image of a honeycomb lattice of micropillars with homogeneous hoppings $\left(t_{L}=t_{L}^{\prime}, \beta=1\right.$, see inset). The circles pinpoint the upper surface of the micropillars in two adjacent hexagons; the white arrows indicate the direction along which the coupling $t_{L}^{\prime}$ is modified. (e) Experimental photoluminescence of an unstrained lattice showing $s$ and $p$ bands. 


\section{TILTED AND SEMI-DIRAC CONES}

The dispersion of the $p$ bands can be modified by introducing an artificial uniaxial strain in the lattice. To do so, we change the center-to-center distance $d^{\prime}$ between the micropillars whose link is oriented along the $x$ direction. This method is equivalent to modifying $t_{L}^{\prime}$, defined in the inset of Fig. 2(d), while keeping the other two couplings $t_{L}$ constant [44]. The panels in the middle row of Fig. 3 show the spectra of four different lattices with decreasing strain parameters $\beta \equiv t_{L}^{\prime} / t_{L}$. The value of $\beta$ is extracted from the fit of the tight-binding model (white lines) to the experimental dispersions.

Let us first focus on the type-I Dirac cones in the central region of Fig. 3 (orange dashed rectangle) around $E_{0}$, the onsite energy of $p$ orbitals in a single micropillar. The upper panels depict the calculated dispersion obtained from the tight-binding model. Decreasing $\beta$, that is, emulating the stretching of the lattice, brings the Dirac cones closer together in the $k_{y}$ direction [Figs. 3(a) and 3(b)] until they merge at $\beta=0.5$ in a single band touching [Fig. 3(c)]. For $\beta<0.5$, a gap is opened [Figs. 3(d) and S2 in Ref. [41]]. This case is a topological Lifshitz transition in which two Dirac cones with opposite topological charge merge and annihilate, as predicted for standard $s$ band graphene $[4,45]$ and reported in photonic $[7,8]$ and atomic $[5,6]$ honeycomb lattices and in black phosphorus [9]. At the merging point $[\beta=0.5$, Fig. 3(c)], we provide the direct observation of a semi-Dirac dispersion, with the touching of two parabolic bands along the $k_{y}$ direction and a linear dispersion along $k_{x}$ [shown in the left inset of Fig. 3(c)].

The Dirac points that we just analyzed do not present any tilt: $v_{0 x}=v_{0 y}=0$ for any value of the strain. Tilted type-I Dirac cones become apparent when analyzing the evolution, as a function of strain, of the touching between the quadratic and flat bands at the $\Gamma$ point [green dashed square in Fig. 3(a)]. For decreasing values of $\beta$, the flat band evolves into a dispersive band with negative effective mass at the $\Gamma$ point, and the band touching gives rise to two Dirac points that move away from each other in the $k_{y}$ direction [Figs. 3(b)-3(d)] [46]. Remarkably, they are tilted as indicated by the angle bisector in green dashed lines in Fig. 3(c). A fit of Eq. (2) to the experimental dispersions close to the Dirac points reveals the evolution of $v_{0 y}$ and $v_{y}$ as a function of the strain, as depicted in Fig. 4. The fitting method is detailed in Appendix C. The measured values of the tilt $\left(v_{0 y}\right)$ agree well with those expected from the tightbinding Hamiltonian (Appendix B) shown in solid lines.
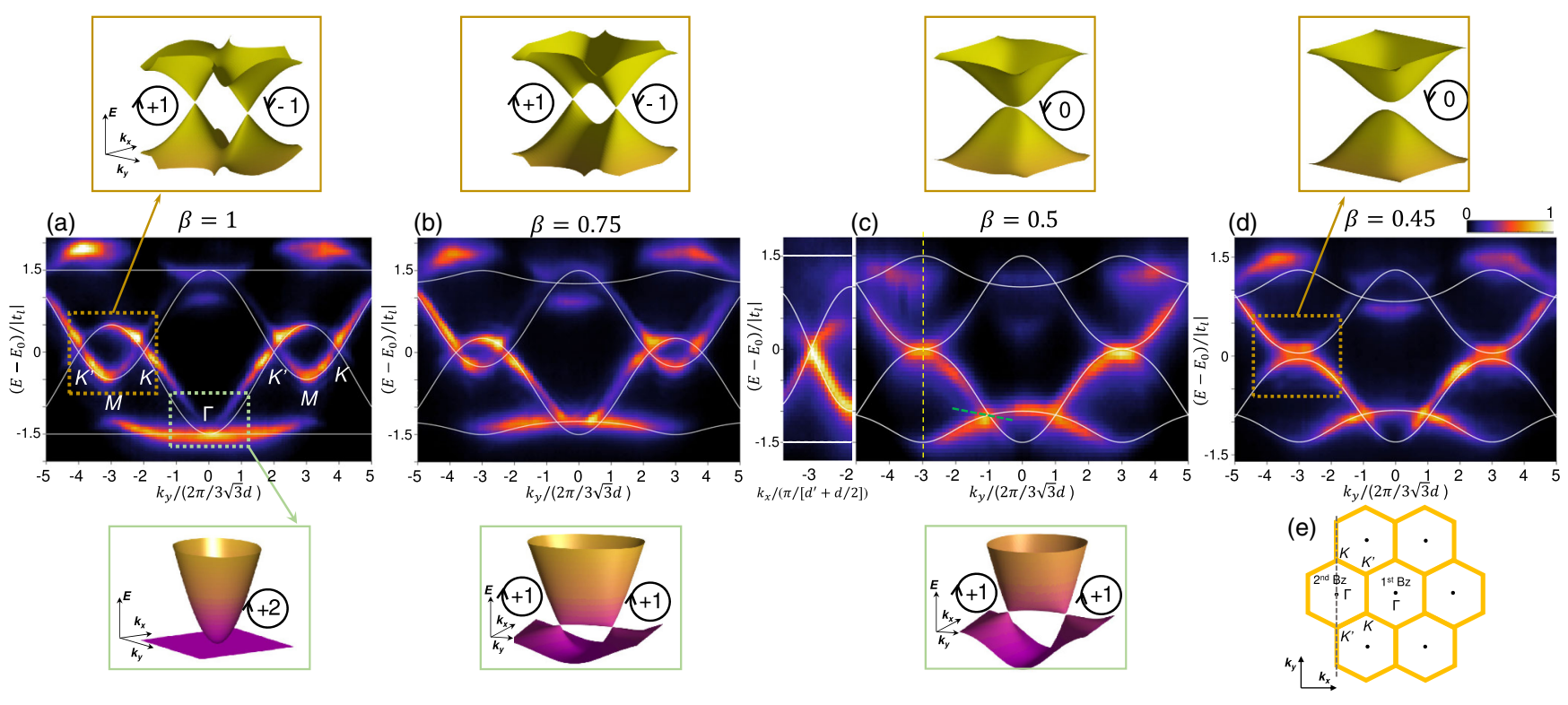

FIG. 3. Tilted Dirac cones in orbital graphene under strain. The central panels (a)-(d) show the measured polariton photoluminescence intensity as a function of $k_{y}$ for different values of $\beta$ (the color scale of each panel has been independently normalized to its maximum value). The plot is done for $k_{x}=2 \pi /\left(d^{\prime}+d / 2\right)$, dashed line in (e). Note that $d=2.40 \mu \mathrm{m}$ for the unstrained coupling, while for the strained coupling, $d^{\prime}$ is $2.40 \mu \mathrm{m}, 2.60 \mu \mathrm{m}, 2.70 \mu \mathrm{m}$, and $2.72 \mu \mathrm{m}$, respectively, in (a)-(d). Here, $E_{0}=1.5687 \mathrm{eV}$ and photon-exciton detuning $\delta=-10 \mathrm{meV}$ at the energy minimum of the $p$ bands for panels (a), (b), and (d); $E_{0}=1.5780 \mathrm{eV}$ and $\delta=-2 \mathrm{meV}$ for panel (c); $t_{L}=-0.90 \mathrm{meV}$ for panel (a), $-0.85 \mathrm{meV}$ for panels (b) and (d), and $-0.65 \mathrm{meV}$ for panel (c), which are obtained by fitting the measured spectra with a tight-binding Hamiltonian (fits are shown as white lines). The left inset of panel (c) shows the semi-Dirac dispersion measured along $k_{x}$ for the value of $k_{y}$ marked by a yellow dashed line in panel (c). The upper row in panels (a)-(d) depicts the calculated tight-binding bands for energies close to $E_{0}$ [orange dashed rectangle in (a)] and the winding around the Dirac points. The bottom row shows the tight-binding bands in the region at the bottom of the bands [green dashed rectangle in (a)] together with the windings. (e) Sketch of the Brillouin zones in momentum space. 


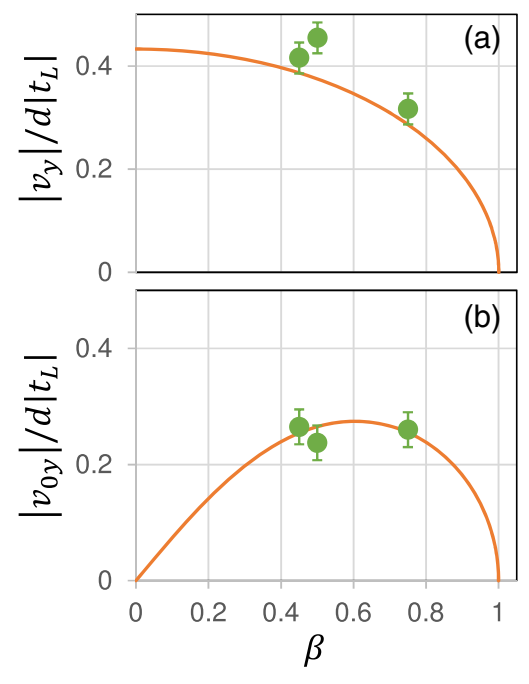

FIG. 4. Dirac cone tilt. Green dots: Absolute value of $v_{y}$ (a) and $v_{0 y}$ (b) extracted from fits of Eq. (2) to the two experimental Dirac cones visible at low energies in Figs. 3(b)-3(d) (see Appendix C). The plotted value is the average of $\left|v_{y}\right|$ and $\left|v_{0 y}\right|$ extracted from the two Dirac points. Solid lines show the tight-binding result.

\section{ENGINEERING TYPE-III DIRAC CONES}

Critically tilted type-III Dirac cones with $\tilde{v}_{0}=$ $\sqrt{\left(v_{0 x} / v_{x}\right)^{2}+\left(v_{0 y} / v_{y}\right)^{2}}=1$ can be implemented in our system when instead of expanding the lattice $(\beta<1)$, it is compressed $(\beta>1)$. The result of the compression in the polariton lattice is shown in Fig. 5(c) for $\beta=1.5$ : In the direction $k_{x}$ parallel to that along which the center-to-center distance $d^{\prime}$ is reduced, two new Dirac points emerge from the flat-parabolic band touching. As a reference, Fig. 5(a) shows the dispersion along $k_{x}$ for $\beta=1$. The most striking feature of the new Dirac points is that they show the crossing of a flat band with zero group velocity and a linear band with finite group velocity [see Fig. 5(c), dashed rectangle]. This feature is precisely the signature of a type-III Dirac cone, and it implies $v_{0 x}=-v_{x}$. From the experimental photoluminescence of Fig. 5(c), we measure $\left|v_{0 x}\right|=(0.29 \pm 0.03) \bar{d}\left|t_{L}\right|$ and $\left|v_{x}\right|=(0.35 \pm$ $0.03) \bar{d}\left|t_{L}\right| \quad\left[\right.$ with $\bar{d}=\left(2 d^{\prime}+d\right) / 3$ ], which is in good agreement with the tight-binding prediction for $\beta=1.5$ $\left(\left|v_{0 x}\right|=\left|v_{x}\right|=0.37 \bar{d}\left|t_{L}\right|\right.$, see Appendix C). Along the perpendicular direction $\left[k_{y}\right.$, Fig. $\left.5(\mathrm{~d})\right]$, the type-III cones present a symmetric linear crossing [Fig. 5(e)], and we measure $\left|v_{0 y}\right|=(0.00 \pm 0.04) d\left|t_{L}\right|,\left|v_{y}\right|=(0.46 \pm 0.04) d\left|t_{L}\right|$, in agreement with the tight-binding result $\left(0\right.$ and $0.48 d\left|t_{L}\right|$, respectively).

The appearance of a flat band implies the formation, in real space, of localized plaquette states decoupled from each other. A well-known example is the hexagonal plaquette states associated with the two-dimensional flat band of a Kagome lattice [47]. In the type-III Dirac cone, the flat band is one-dimensional along $k_{x}$ with $k_{y}=0$

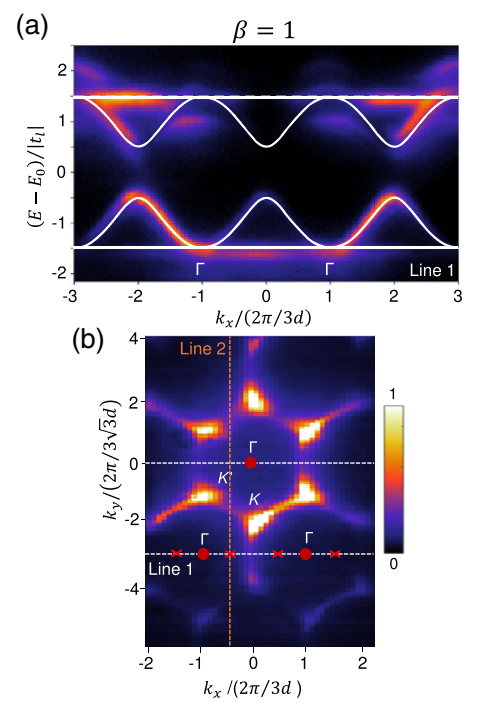

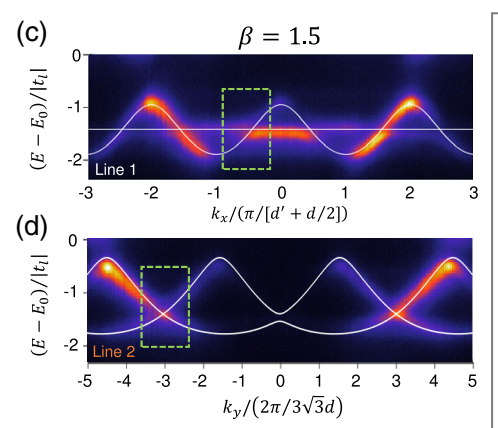

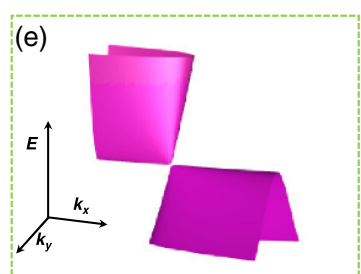

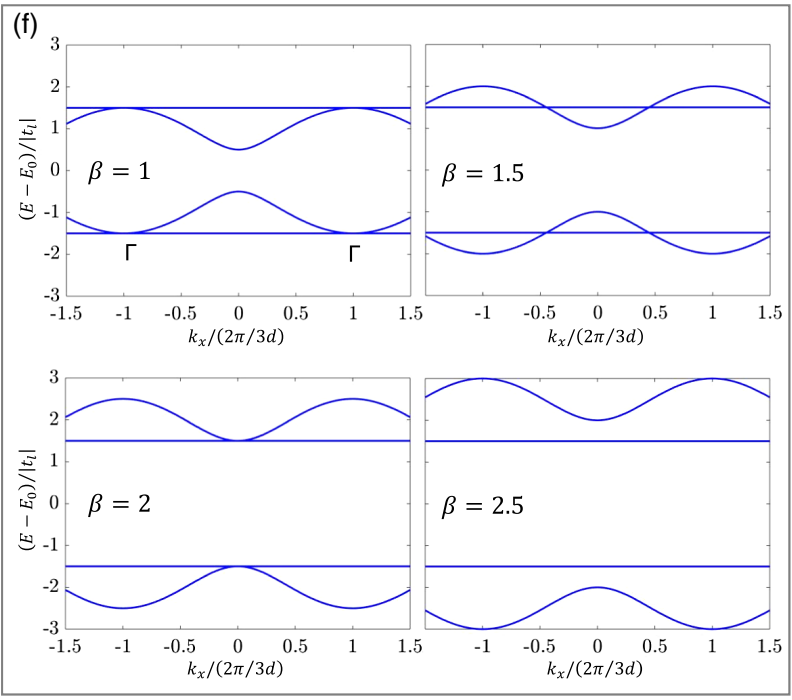

FIG. 5. Type-III Dirac cones. (a) Measured photoluminescence intensity for $\beta=1$ (micropillar diameter: $2.60 \mu$ m, $\left.d=d^{\prime}=2.40 \mu \mathrm{m}\right)$ along $k_{x}$ for $k_{y}=-2 \pi /(\sqrt{3} d)$ [line 1 in panel (b)]. Here, $E_{0}=1.5687 \mathrm{eV}$ and $t_{L}=-0.85 \mathrm{meV}$. (b) Emission in momentum space at $E_{0}$ for $\beta=1$, showing the usual Dirac points at the high-symmetry points in reciprocal space. Crosses indicate the position of the type-III Dirac points reported in panel (c). (c) Zoom of the low-energy section of the measured spectrum for a lattice with $\beta=1.5\left(d=2.40 \mu \mathrm{m}, d^{\prime}=2.10 \mu \mathrm{m}, t_{L}=-0.78 \mathrm{meV}\right)$ showing the emergence of type-III Dirac cones combining flat and dispersive bands. (d) Measured dispersion along the $k_{y}$ direction for $k_{x}=-(0.5 \times 2 \pi) / 3 d$ [line 2 in panel (b)], crossing the Dirac point in the dashed rectangle in panel (c). (e) Dispersion obtained from the tight-binding model zoomed in the momentum space region marked with a dashed rectangle in panels (c) and (d). In panels (a), (c), and (d), the white lines show the tight-binding model. (f) Calculated dispersions for $k_{y}=-2 \pi /(\sqrt{3} d)$ for different values of $\beta>1$. Type-III Dirac cones merge at $k_{x}=0$ for $\beta=2$. 
(a) Type-III Dirac cone

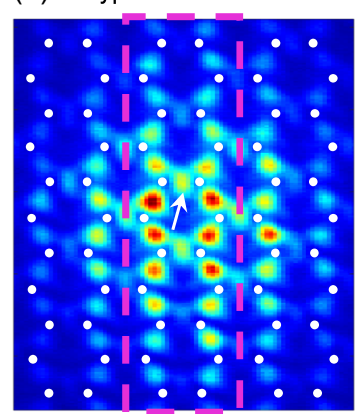

(c) Simulation type-III

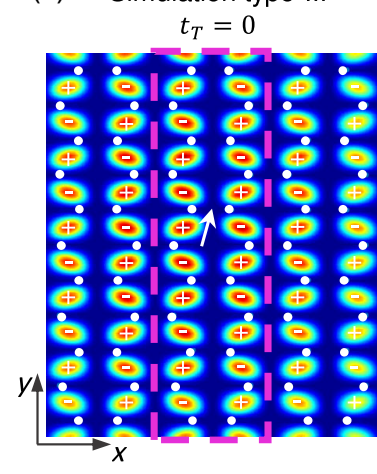

(b) Top of $s$ bands

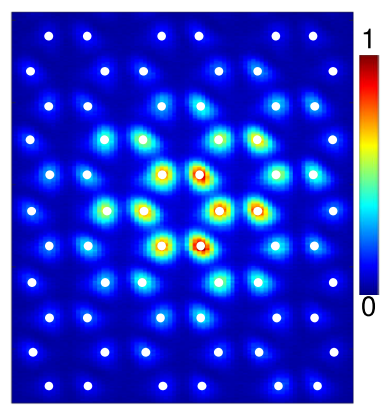

(d) Simulation type-III $t_{T}=-0.1 t_{L}$

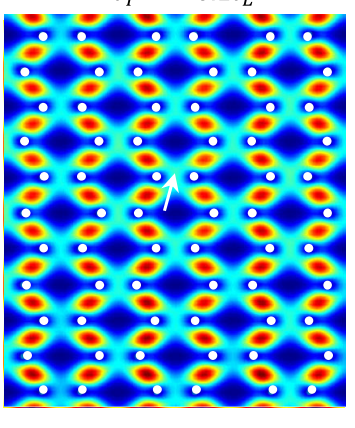

FIG. 6. Real-space wave functions of the flat band at the typeIII Dirac cones. (a) Measured real-space photoluminescence intensity for $\beta=1.5$ at the energy of the type-III Dirac cone in Fig. 5(c) $(E=1.56752 \mathrm{eV})$. The white arrow points to the emission observed in between the horizontal links. (b) Same for the top of the $s$ bands $(E=1.56577 \mathrm{eV})$, for which the usual hexagonal emission is observed (here shown as a reference). The white dots mark the center of the micropillars. (c) Calculated eigenstate at the energy of the flat band at the $\Gamma$ point with $t_{T}=0$. To obtain the real-space distribution, we account for the $p_{x}$ and $p_{y}$ shapes of the orbitals in each micropillar. The \pm signs indicate the sign of the wave function in each lobe. In panels (a) and (c), the dashed rectangles mark the shape of a plaquette state. (d) Same as panel (c) with $t_{T}=-0.1 t_{L}$. The arrows highlight one of the strained links.

[equivalently, $k_{y}=-2 \pi /(\sqrt{3} d)$, in the next Brillouin zone]. Figure 6(c) shows the calculated wave function for the $k_{x}=$ $k_{y}=0$ state of the flat band for $\beta=1.5$ (blue dots show the position of the center of the micropillars). We can see that this state only contains $p_{y}$ orbitals, which couple the micropillars in the vertical direction, and that $p_{x}$ orbitals are absent. Indeed, it can be easily shown that for $k_{y}=0, p_{x}$ and $p_{y}$ orbitals are decoupled [41], and the flat-band states are onedimensional plaquettes running along the vertical direction and decoupled from the adjacent plaquettes located at their sides [dashed rectangles in Figs. 6(a) and 6(c) show one plaquette]. Similar one-dimensional plaquette states have recently been reported related to the two-dimensional flat band of a Lieb lattice [48].

Figure 6(a) shows the real-space emission measured at the energy of the type-III Dirac cone reported in
Figs. 5(c)-5(d), with an excitation spot of diameter $8.4 \mu \mathrm{m}$ (full width at half maximum). One can recognize the high-intensity lobes aligned as expected for the plaquette states. In addition, a fainter emission can be seen in between the horizontal links (a white arrow highlights one of these links). The reason is that in the experiment, the coupling of $p$ modes with lobes oriented perpendicular to the links is not strictly zero $\left(t_{T} \neq 0\right)$. This is particularly true for the lattices showing the type-III Dirac cone, in which the horizontal links have been made shorter to implement a value of strain of $\beta=1.5$. Such faint emission between the micropillars can be reproduced by simulations [Fig. 6(d), see black arrow] when including hopping between transverse orbitals $t_{T}=-0.1 t_{L}$ on the strained links.

The decoupling of $p_{x}$ and $p_{y}$ orbitals at $k_{y}=0$ explains why the one-dimensional flat band is always present in the $p$ bands no matter the value of $\beta$ [see Fig. 5(f)]. Indeed, the change of $\beta$ only affects the coupling between $p_{x}$ orbitals in the strained links, and it does not affect the flat band that emerges exclusively from the coupling between $p_{y}$ orbitals. This is also the reason for the extraordinary robustness of the type-III Dirac cone against perturbations of the hopping parameters in the lattice. The type-III cones do not arise from the gradual tilting of type-I Dirac cones. Instead, they emerge from the deformation of the dispersive band that touches the flat band when the uniaxial synthetic strain is increased. Figure 5(f) shows the evolution of the bands according to the tight-binding model as a function of $\beta$ : The energy of the flat band at $k_{y}=0$ remains constant when varying $\beta$, while the dispersive bands are pushed towards lower and higher energies. The type-III Dirac cones are thus present for all values of $\beta$ between 1 and 2, proving their robustness against variations of the strain parameter.

\section{TOPOLOGICAL INVARIANTS OF TILTED AND TYPE-III DIRAC CONES}

The emergence of unconventional Dirac cones when applying a uniaxial deformation to the orbital lattice can be understood from topological arguments. The analysis of the topological charge associated with each cone, that is, the winding of the Hamiltonian around each Dirac point, provides a clear picture of their birth and evolution. The vicinity of the parabolic-flat-band touching point at the $\Gamma$ point for $\beta=1$ can be described by an effective $2 \times 2$ Hamiltonian obtained from the projection of the full $4 \times 4$ tight-binding Hamiltonian on the subspace of the two lowest-energy bands [49], resulting in the following effective Hamiltonian:

$$
\begin{aligned}
H(\mathbf{q}) & =-\frac{3}{2}\left|t_{L}\right| \sigma_{0}+\frac{3}{8}\left|t_{L}\right|\left(\begin{array}{cc}
q_{x}^{2} & q_{x} q_{y} \\
q_{x} q_{y} & q_{y}^{2}
\end{array}\right) \\
& \equiv-\frac{3}{2}\left|t_{L}\right| \sigma_{0}+\mathbf{h}(\mathbf{q}) \cdot \boldsymbol{\sigma} .
\end{aligned}
$$


Matrix elements coupling the high- and low-energy manifolds are treated in a second-order perturbation theory [49]. The winding of $\mathbf{h}(\mathbf{q})$ takes the form of a winding vector [45]: $\mathcal{W}=(2 \pi)^{-1} \oint \mathbf{n}(\mathbf{q}) \times \mathbf{d n}(\mathbf{q})$, where $\mathbf{n}(\mathbf{q})=\mathbf{h}(\mathbf{q}) /|\mathbf{h}(\mathbf{q})|$, and the integral is performed along a closed line in momentum space that encircles the band touching point [41]. The modulus of $\mathcal{W}$ is always an integer, thus providing a topological charge to the touching point, and it has a value $\mathcal{W}=2$ in this case.

We can extend this analysis to the emerging Dirac cones when $\beta \neq 1$. Following the same procedure, the tight-binding Hamiltonian can be reduced to an effective $2 \times 2$ matrix close to the considered Dirac cone. For $\beta<1$, the energy of the Dirac cones in the lower part of the spectrum is $E_{D}=E_{0}-\frac{1}{2}\left|t_{L}\right| \sqrt{3+6 \beta^{2}}$. Taking $E_{D}$ as the origin of energies, the effective Hamiltonian near a Dirac point reads:

$$
H(\mathbf{q})=v \cos \theta q_{y} \sigma_{0}+v q_{y} \sigma_{\theta}+v \cos \theta q_{x} \sigma_{x},
$$

where $v=(\sqrt{3} / 4) d\left|t_{L}\right| \sqrt{\left|1-\beta^{2}\right|}$, the angle $\theta \in[0, \pi / 2]$ is defined as $\tan \theta=\sqrt{1-\beta^{2}} / \sqrt{3} \beta$ (with $\theta \rightarrow \pi-\theta$ for the other Dirac point), and $\sigma_{\theta}=\boldsymbol{\sigma} \cdot \boldsymbol{u}_{\theta}$, with $\boldsymbol{u}_{\theta}=$ $-\sin \theta \boldsymbol{u}_{y}+\cos \theta \boldsymbol{u}_{z}$, where $\boldsymbol{u}_{x, y, z}$ are Cartesian unit vectors. Analogously, for $\beta>1$, around a type-III Dirac point $\left(E_{D}=E_{0}-\frac{3}{2}\left|t_{L}\right|\right)$, the reduced Hamiltonian reads

$$
H(\mathbf{q})=v \cos \phi q_{x} \sigma_{0}+v q_{y} \sigma_{\phi}-v \cos \phi q_{x} \sigma_{z},
$$

where $\tan \phi=\sqrt{\beta^{2}-1} / \sqrt{4-\beta^{2}}$ and $\sigma_{\phi}=\boldsymbol{\sigma} \cdot \boldsymbol{u}_{\phi}$, with $\boldsymbol{u}_{\phi}=\cos \phi \boldsymbol{u}_{x}-\sin \phi \boldsymbol{u}_{y}$ (for the other Dirac point, $\phi \rightarrow \pi-\phi)$. By comparing $H(\mathbf{q})$ in Eqs. (4) and (5) with the generalized Dirac Hamiltonian [Eq. (1)], we can directly extract the Dirac effective velocities as a function of $\beta$ (shown as solid lines in Fig. 4 for $v_{0 y}$ and $v_{y}$ ) as well as the tilt parameter $\tilde{v}_{0}$ :

\begin{tabular}{cccccc}
\hline & $\left|v_{0 x}\right|$ & $\left|v_{0 y}\right|$ & $\left|v_{x}\right|$ & $\left|v_{y}\right|$ & $\tilde{v}_{0}$ \\
\hline$\beta<1$ & 0 & $v \cos \theta$ & $v \cos \theta$ & $v$ & $\cos \theta$ \\
$\beta>1$ & $v \cos \phi$ & 0 & $v \cos \phi$ & $v$ & 1 \\
\hline
\end{tabular}

To compute the winding associated with the Dirac cones, Hamiltonians (4) and (5) can be rearranged similarly to the right-hand side of Eq. (3), with a term in the form $\mathbf{h}(\mathbf{q}) \cdot \boldsymbol{\sigma}$. For $\beta<1$ [Hamiltonian (4)], the winding of $\mathbf{h}(\mathbf{q})$ around each of the Dirac cones is $\mathcal{W}=1$, as indicated on the lower panels of Figs. 3(b) and 3(c). This is also the case for the type-III Dirac cones for $\beta>1$ [winding of Hamiltonian (5)].

A prominent feature of these Hamiltonians is that the vector $\mathbf{h}(\mathbf{q})$ winds on a plane that depends on the deformation $\beta$. Namely, for $\beta<1$, the pseudofield $\mathbf{h}(\mathbf{q})$ lives in the plane $\left(\boldsymbol{u}_{x}, \boldsymbol{u}_{\theta}\right)$, and the winding vector points in
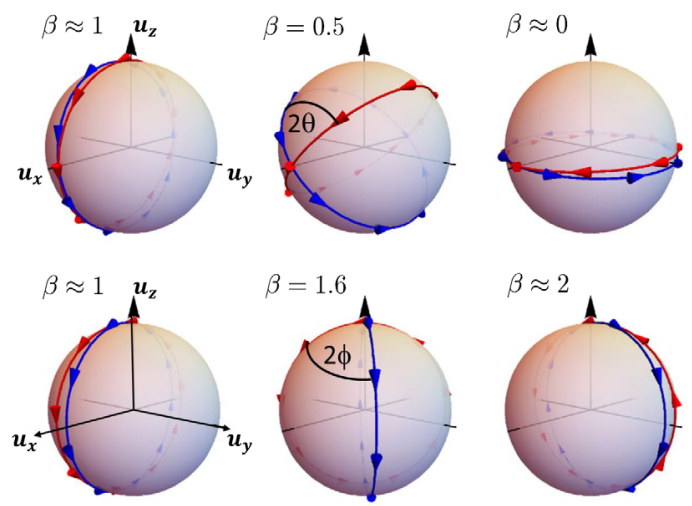

FIG. 7. Evolution of the winding plane around the tilted Dirac cones. The arrows indicate the direction of $\mathbf{h}(\mathbf{q})$ as a function of $\beta$ around the two Dirac cones (red and blue lines, respectively) emerging from the parabolic-flat-band touching.

the direction $\mathcal{W}=(0, \cos \theta, \pm \sin \theta)$, where the \pm sign corresponds to each of the two Dirac cones. The winding vector plane thus rotates as a function of $\beta$. As each of the two emergent Dirac cones is described by an angle $\theta$ of opposite sign, the winding plane turns in opposite directions for each Dirac cone when $\beta$ goes to zero. This case is illustrated by the red and blue circles in Fig. 7, which show, respectively, the winding plane of each of the two Dirac cones. This is very different from regular $s$ band graphene, in which the vector $\mathbf{h}(\mathbf{q})$ resides in the plane $\left(\boldsymbol{u}_{x}, \boldsymbol{u}_{y}\right)$ no matter the value of the strain $\beta$ [4].

For $\beta>1$, the vector $\mathbf{h}(\mathbf{q})$ resides in the plane $\left(\boldsymbol{u}_{\phi}, \boldsymbol{u}_{z}\right)$, and the winding vector points in the direction $\mathcal{W}=( \pm \sin \phi, \cos \phi, 0)$. Note that at $\beta=2$ (bottom-right panel in Fig. 7), the two emergent Dirac cones are described by a winding vector residing in the same plane but pointing in opposite directions. Therefore, the winding around each Dirac cone has opposite sign: $\mathcal{W}=+1$ and $\mathcal{W}=-1$. At $\beta=2$, actually, the Dirac cones merge at the $\mathrm{M}$ point and annihilate, opening a gap for $\beta>2$. This situation is clearly seen in the tight-binding calculation shown in Fig. 5(f), and it could not be observed experimentally because the engineered hoppings in the compressed structures were limited to $\beta=1.5$. This kind of merging preceded by a rotation of the winding plane has recently been discussed in detail in the context of the Mielke lattice under strain [45].

The analysis of the windings sheds light on the mechanisms behind the creation of Dirac cones starting from a flat band touching a dispersive band. The single band touching at $\beta=1$ is described by a winding $\mathcal{W}=2$. When $\beta \gtrless 1$, the band touching evolves into a pair of Dirac cones (tilted type-I or type-III) with winding $\mathcal{W}=1$. This case is an illustration of one of the two possible scenarios for Dirac merging in two dimensions, characteristic of, for example, bilayer graphene [46]. Remarkably, the orbital $p$ bands show the two possible universal scenarios for the merging 
of Dirac cones in two dimensions [50]: (i) Two Dirac cones with $\mathcal{W}=1$ emerge from a point with $\mathcal{W}=2$, which we have just discussed, and it is reported in the lower part of the spectra of Fig. 3; (ii) two Dirac cones with opposite winding $\mathcal{W}= \pm 1$ merge in a semi-Dirac cone with $\mathcal{W}=0$, reported in the spectra in the central row of Fig. 3 (a $2 \times 2$ effective Hamiltonian analysis can also be done for the Dirac points at $E_{0}$ ) and for the type-III Dirac cones at $\beta=2$ [Fig. 5(f)].

\section{CONCLUSION}

The photonic realization reported here demonstrates the flexibility of orbital bands to implement unconventional Dirac points. This is an asset for the engineering of photonic materials that combine different types of Dirac dispersions, a promising configuration for the study of analogue black holes in photonics $[21,25,26]$. Moreover, our experiments provide a recipe for the implementation of Dirac cones in solid-state materials: The touching of a flat and a dispersive band with winding $\mathcal{W}=2$ evolves into two Dirac cones in the presence of strain. This behavior has been predicted for other lattice geometries [45], and it presents a natural playground to investigate the transition between different topological phases when particle interactions are present $[51,52]$ or when time-reversal symmetry is broken. Polaritons are particularly well suited to study these scenarios: Thanks to their excitonic component, they present significant repulsive interactions in the high-density regime, which can be achieved when using a resonant excitation scheme [53]; they are also sensitive to external magnetic fields, allowing the implementation of quantum Hall phases [54,55]. Polariton orbital bands of the kind reported here open exciting perspectives for the study of topological lasers [56,57] and of Dirac superfluids in the mean-field interacting regime [58]. Going a step further, recent experimental reports have shown evidence of singlepolariton blockade effects in a single confined site $[59,60]$, demonstrating the great potentiality of the polariton platform to study strongly correlated phases in elaborate landscapes such as those provided by orbital polariton lattices.

\section{ACKNOWLEDGMENTS}

This work was supported by the ERC grant Honeypol, the EU-FET Proactive grant AQuS, the Quantera grant Inerpol, the FETFLAG grant PhoQus, the French National Research Agency (ANR) project Quantum Fluids of Light (ANR-16CE30-0021), the Labex CEMPI (ANR-11-LABX-0007) and NanoSaclay (ICQOQS, Grant No. ANR-10-LABX-0035), the French RENATECH network, the CPER Photonics for Society P4S, the I-Site ULNE via the project NONTOP and the Métropole Européenne de Lille via the project TFlight. T. O. acknowledges support from JSPS KAKENHI Grant No. JP18H05857, RIKEN Incentive Research Project, and the Interdisciplinary Theoretical and Mathematical Sciences Program (iTHEMS) at RIKEN.

\section{APPENDIX A: SAMPLE DESCRIPTION}

The sample used in the experiments is a semiconductor microcavity grown by molecular beam epitaxy. Two Bragg mirrors made of 28 (top) and 40 (bottom) pairs of $\lambda / 4$ alternating layers of $\mathrm{Ga}_{0.05} \mathrm{Al}_{0.95} \mathrm{As} / \mathrm{Ga}_{0.80} \mathrm{Al}_{0.20} \mathrm{As}$ embed a $\lambda / 2 \mathrm{Ga}_{0.80} \mathrm{Al}_{0.20}$ As cavity. Here, $\lambda=775 \mathrm{~nm}$ is the resonance wavelength of the free excitons in the twelve GaAs, 7-nm-wide quantum wells grown in groups of four in the three central maxima of the electromagnetic field of the cavity. At $10 \mathrm{~K}$, the temperature of the experiments, the excitonic and photonic resonances are in the strong coupling regime, with a Rabi splitting of $15 \mathrm{meV}$. The experiments are performed at a photon-exciton detuning of $-10 \mathrm{meV}$ (measured at the energy of the lowest-energy flat band), except for the data shown in Fig. 3(c), for which the detuning is $-2 \mathrm{meV}$.

To fabricate the honeycomb lattices, the as-grown planar structure is subject to e-beam lithography and inductively coupled plasma etching down to the GaAs substrate. Each micropillar in the lattice has a diameter of $2.75 \mu \mathrm{m}$, and the center-to-center distance varies between 2.40 and $2.72 \mu \mathrm{m}$, ensuring the overlap between adjacent micropillars. For the observation of the type-III Dirac cones (Figs. 5 and 6), the micropillar diameter is $2.60 \mu \mathrm{m}$, and the center-to-center distance varies from 2.40 to $2.10 \mu \mathrm{m}$. The polariton lifetime measured in a similar unetched microcavity is of the order of $30 \mathrm{ps}$. In the etched structures, the lateral defects induced during the microfabrication process reduce the lifetime to about $5-15$ ps at the considered photon-exciton detunings.

\section{APPENDIX B: TIGHT-BINDING HAMILTONIAN AND EXTRACTION OF $\beta$}

The coupling of the $p_{x}, p_{y}$ orbitals in the isotropic honeycomb lattice can be described by a tight-binding Hamiltonian of the form $[36,43]$

$$
\begin{aligned}
H & =\sum_{m, n}-\left[t_{L}\left(\hat{a}_{m, n}^{\dagger} \cdot \mathbf{e}_{L 1}\right)\left(\mathbf{e}_{L 1} \cdot \hat{b}_{m, n}^{\dagger}\right)\right. \\
& +t_{L}\left(\hat{a}_{m+1, n}^{\dagger} \cdot \mathbf{e}_{L 2}\right)\left(\mathbf{e}_{L 2} \cdot \hat{b}_{m, n}^{\dagger}\right) \\
& +t_{L}\left(\hat{a}_{m, n+1}^{\dagger} \cdot \mathbf{e}_{L 3}\right)\left(\mathbf{e}_{L 3} \cdot \hat{b}_{m, n}^{\dagger}\right) \\
& +t_{T}\left(\hat{a}_{m, n}^{\dagger} \cdot \mathbf{e}_{T 1}\right)\left(\mathbf{e}_{T 1} \cdot \hat{b}_{m, n}^{\dagger}\right) \\
& +t_{T}\left(\hat{a}_{m+1, n}^{\dagger} \cdot \mathbf{e}_{T 2}\right)\left(\mathbf{e}_{T 2} \cdot \hat{b}_{m, n}^{\dagger}\right) \\
& \left.+t_{T}\left(\hat{a}_{m, n+1}^{\dagger} \cdot \mathbf{e}_{T 3}\right)\left(\mathbf{e}_{T 3} \cdot \hat{b}_{m, n}^{\dagger}\right)+\text { H.c. }\right],
\end{aligned}
$$

where $\mathbf{e}_{L 1, L 2, L 3}$ are unit vectors oriented parallel to the nearest-neighbor links of each site [see Fig. 2(c)]; $\mathbf{e}_{T 1, T 2, T 3}$ are unit vectors oriented perpendicular to $\mathbf{e}_{L 1, L 2, L 3}$, respectively, $\hat{a}_{m, n}^{\dagger}=\left(\hat{a}_{m, n(x)}^{\dagger}, \hat{a}_{m, n(y)}^{\dagger}\right)$ and $\hat{b}_{m, n}^{\dagger}=\left(\hat{b}_{m, n(x)}^{\dagger}, \hat{b}_{m, n(y)}^{\dagger}\right)$, with $\hat{a}_{m, n(x, y)}^{\dagger}, \hat{b}_{m, n(x, y)}^{\dagger}$ the creation operators of a photon in the $p_{x}$ or $p_{y}$ orbital of the $a, b$ site of the $m, n$ unit 
cell in the lattice. If the hopping of orbitals oriented perpendicular to the links is negligible $\left(t_{T}=0\right)$, Hamiltonian (B1) can be written in momentum space in the form [43]

$H(\mathbf{k})=-t_{L}\left(\begin{array}{cc}0_{2 \times 2} & Q(\mathbf{k}) \\ Q(\mathbf{k})^{\dagger} & 0_{2 \times 2}\end{array}\right), \quad Q(\mathbf{k})=\left(\begin{array}{cc}f_{1} & g \\ g & f_{2}\end{array}\right)$,

where we have assumed that the on-site energy for the $p_{x}, \quad p_{y}$ orbitals is $0, f_{1}=\frac{3}{4}\left(e^{i \mathbf{k} \mathbf{a}_{1}}+e^{i \mathbf{k} \mathbf{a}_{2}}\right), f_{2}=1+$ $\frac{1}{4}\left(e^{i \mathbf{k} \mathbf{a}_{1}}+e^{i \mathbf{k} \mathbf{a}_{2}}\right)$, and $g=(\sqrt{3} / 4)\left(e^{i \mathbf{k} \mathbf{a}_{1}}-e^{i \mathbf{k} \mathbf{a}_{2}}\right)$, with $\mathbf{a}_{1}=$ $\left[\frac{3}{2} d,(\sqrt{3} / 2) d\right]$ and $\mathbf{a}_{2}=\left[\frac{3}{2} d,-(\sqrt{3} / 2) d\right]$ the lattice vectors depicted in Fig. 2(c) and $\mathbf{k}=\left(k_{x}, k_{y}\right)$ the quasimomentum. The diagonalization of Hamiltonian (B2) results in four bands depicted as solid white lines in Fig. 3(a). The spectrum shows two flat bands at high and low energy that touch two dispersive bands with two nonequivalent Dirac points at $E=0$.

If we account for different hoppings $t_{L}$ and $t_{L}^{\prime}$ for different links, Hamiltonian (B2) is still valid with the same expressions for $f_{1}$ and $g$, and $f_{2}$ replaced by $f_{2}=\beta+\frac{1}{4}\left(e^{i \mathbf{k} \mathbf{a}_{1}}+e^{i \mathbf{k} \mathbf{a}_{2}}\right)$. As a function of $\beta=t_{L}^{\prime} / t_{L}$, its spectrum $\epsilon(\mathbf{k})$ takes the form

$$
\epsilon(\mathbf{k})= \pm \sqrt{E^{\prime}(\mathbf{k}) \pm E(\mathbf{k})},
$$

where the four sign combinations correspond to the four bands, and

$$
\begin{aligned}
E(\mathbf{k})= & \left|t_{L}\right|\left[\frac { 3 } { 4 } \operatorname { s i n } ^ { 2 } ( K _ { y } ) \left(\beta^{2}+\cos ^{2}\left(K_{y}\right)\right.\right. \\
& \left.-2 \beta \cos \left(K_{x}\right) \cos \left(K_{y}\right)\right)+\frac{1}{4}\left(2 \cos ^{2}\left(K_{y}\right)\right. \\
& \left.\left.-\beta^{2}-\beta \cos \left(K_{x}\right) \cos \left(K_{y}\right)\right)^{2}\right]^{1 / 2}, \\
E^{\prime}(\mathbf{k})= & \frac{\left|t_{L}\right|}{4}\left[3+2 \beta^{2}+2 \cos ^{2}\left(K_{y}\right)+2 \beta \cos \left(K_{x}\right) \cos \left(K_{y}\right)\right],
\end{aligned}
$$

with $K_{x} \equiv \frac{3}{2} k_{x} d$ and $K_{y} \equiv(\sqrt{3} / 2) k_{y} d$.

For $\beta<1$, the energy $E_{D}$ and momentum-space coordinates of the Dirac points emerging at the bottom bands are, respectively,

$E_{D}=E_{0}-\frac{\left|t_{L}\right|}{2} \sqrt{3+6 \beta^{2}}, \quad K_{x}=0, \quad \cos \left(K_{y}\right)=\beta$,

where $E_{0}$ is the on-site energy, while for $\beta>1$, they are
$E_{D}=E_{0}-\frac{3}{2}\left|t_{L}\right|, \quad \cos \left(K_{x}\right)=\frac{2-\beta^{2}}{\beta}, \quad K_{y}=0$.

In Figs. 3 and 5, the experimental value of $\beta$ is obtained from fits of the tight-binding model with $t_{T}=0$ to the measured dispersions $\left(E_{0}, t_{L}\right.$ and $\beta$ are the fitting parameters).

\section{APPENDIX C: MEASUREMENT AND CALCULATION OF THE EFFECTIVE DIRAC VELOCITIES}

Figure 4 shows the effective Dirac velocities $v_{0 y}$ and $v_{y}$ measured from the dispersions along the $k_{y}$ direction plotted in Fig. 3. To measure these velocities, we first extract the experimental dispersion around the Dirac cones from the maxima of the photoluminescence intensity as a function of energy and momentum. For the case of $\beta=0.45$, the data points are shown in Fig. 8(b). A linear fit allows measuring the slope of the dispersion. By comparison with Eq. (1), the two slopes correspond to $\left|v_{0 y}\right|-\left|v_{y}\right|$ and $\left|v_{0 y}\right|+\left|v_{y}\right|$. From this result, we extract $\left|v_{0 y}\right|$ and $\left|v_{y}\right|$. We repeat the procedure for the symmetric Dirac point, and we take the average of their absolute values.

Note that for type-I Dirac cones, $v_{0 x}=v_{0 y}=0$, and the absolute value of the two slopes should be identical and equal to $v_{y}$. To test this hypothesis, we have measured the slope around the type-I Dirac cone at $E_{0}$ in Fig. 3(a) at positive momenta. After converting the slopes to effective Weyl velocities [Eq. (1)], we obtain $\left|v_{0 y}\right|=0.07 \pm 0.03$, close to the expected value of 0 , and $\left|v_{y}\right|=0.72 \pm 0.03$ in units of $d\left|t_{L}\right|$.

From the tight-binding Hamiltonian, the effective Dirac velocities can be obtained from the derivative along $k_{x}$ and $k_{y}$ of the eigenvalues of Eqs. (B3)-(B5) at the position of the emergent Dirac cones [Eqs. (B6) and (B7)], resulting in the analytical expressions for $\beta<1$ (normalized to $d\left|t_{L}\right|$, where we have assumed that, in the experiment, $d \approx d^{\prime}$ ):
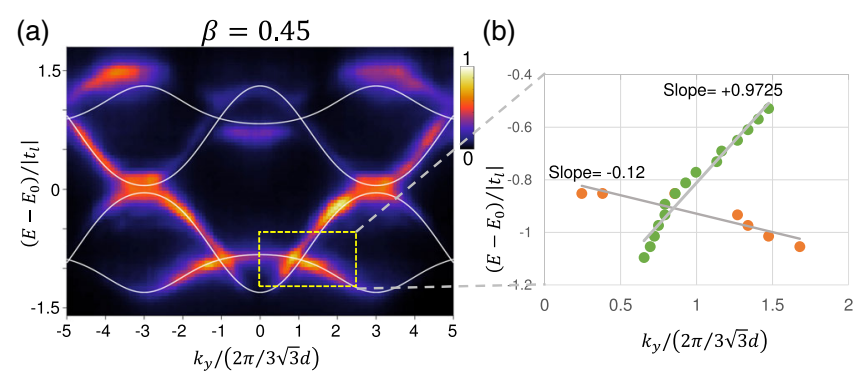

FIG. 8. (a) Measured dispersion along the $k_{y}$ direction for $k_{x}=$ $2 \pi /\left(d^{\prime}+d / 2\right)$ for $\beta=0.45$ (same as Fig. 3). (b) Data points extracted from the Dirac point marked in a dashed rectangle in panel (a). From linear fits to the points, we obtain $\left|v_{0 y}\right|$ and $\left|v_{y}\right|$, plotted in Fig. 4. 


$$
\begin{aligned}
& \left|v_{0 x}\right|=0, \quad\left|v_{x}\right|=\left|v_{0 y}\right|=\frac{3 \beta}{4} \sqrt{\frac{1-\beta^{2}}{1+2 \beta^{2}}}, \\
& \left|v_{y}\right|=\frac{\sqrt{3}}{4} \sqrt{1-\beta^{2}} .
\end{aligned}
$$

The analytical expressions for $\left|v_{0 y}\right|$ and $\left|v_{y}\right|$ are shown as solid lines in Fig. 4.

For $1<\beta<2$, we have (same units as above)

$$
\begin{aligned}
& \left|v_{0 x}\right|=\left|v_{x}\right|=\frac{1}{4} \sqrt{\left(\beta^{2}-1\right)\left(4-\beta^{2}\right)}, \\
& \left|v_{0 y}\right|=0, \quad\left|v_{y}\right|=\frac{\sqrt{3}}{4} \sqrt{\beta^{2}-1} .
\end{aligned}
$$

Note that the fact that $\left|v_{0 x}\right|=\left|v_{x}\right|$ (with opposite sign: $v_{0 x}=-v_{x}$ ) for $\beta>1$ is the signature of the type-III Dirac cone, and it results in a flat band along the $k_{x}$ direction.

[1] A. H. C. Neto, F. Guinea, N. M. R. Peres, K. S. Novoselov, and A. K. Geim, The Electronic Properties of Graphene, Rev. Mod. Phys. 81, 109 (2009).

[2] Y. Zhao, J. Wyrick, F. D. Natterer, J. F. Rodriguez-Nieva, C. Lewandowski, K. Watanabe, T. Taniguchi, L. S. Levitov, N. B. Zhitenev, and J. A. Stroscio, Creating and Probing Electron Whispering-Gallery Modes in Graphene, Science 348, 672 (2015).

[3] B. A. Bernevig and T. L. Hughes, Topological Insulators and Topological Superconductors (Princeton University Press, Princeton, NJ, 2013).

[4] G. Montambaux, F. Piéchon, J.-N. Fuchs, and M. Goerbig, Merging of Dirac Points in a Two-Dimensional Crystal, Phys. Rev. B 80, 153412 (2009).

[5] L.-K. Lim, J.-N. Fuchs, and G. Montambaux, Bloch-Zener Oscillations across a Merging Transition of Dirac Points, Phys. Rev. Lett. 108, 175303 (2012).

[6] L. Tarruell, D. Greif, T. Uehlinger, G. Jotzu, and T. Esslinger, Creating, Moving and Merging Dirac Points with a Fermi Gas in a Tunable Honeycomb Lattice, Nature (London) 483, 302 (2012).

[7] M. Bellec, U. Kuhl, G. Montambaux, and F. Mortessagne, Topological Transition of Dirac Points in a Microwave Experiment, Phys. Rev. Lett. 110, 033902 (2013).

[8] M. C. Rechtsman, Y. Plotnik, J. M. Zeuner, D. Song, Z. Chen, A. Szameit, and M. Segev, Topological Creation and Destruction of Edge States in Photonic Graphene, Phys. Rev. Lett. 111, 103901 (2013).

[9] J. Kim, S. S. Baik, S. H. Ryu, Y. Sohn, S. Park, B.-G. Park, J. Denlinger, Y. Yi, H. J. Choi, and K. S. Kim, Observation of Tunable Band Gap and Anisotropic Dirac Semimetal State in Black Phosphorus, Science 349, 723 (2015).

[10] M. O. Goerbig, J.-N. Fuchs, G. Montambaux, and F. Piéchon, Tilted Anisotropic Dirac Cones in Quinoid-Type Graphene and $\alpha$-(BEDT-TTF $)_{2} \mathrm{I}_{3}$, Phys. Rev. B 78, 045415 (2008).
[11] H.-Y. Lu, A. S. Cuamba, S.-Y. Lin, L. Hao, R. Wang, H. Li, Y. Zhao, and C. S. Ting, Tilted Anisotropic Dirac Cones in Partially Hydrogenated Graphene, Phys. Rev. B 94, 195423 (2016).

[12] S. Katayama, A. Kobayashi, and Y. Suzumura, PressureInduced Zero-Gap Semiconducting State in Organic Conductor $\alpha$-(BEDT-TTF $)_{2} \mathrm{I}_{3}$ Salt, J. Phys. Soc. Japan 75, 054705 (2006).

[13] M. Hirata, K. Ishikawa, K. Miyagawa, M. Tamura, C. Berthier, D. Basko, A. Kobayashi, G. Matsuno, and K. Kanoda, Observation of an Anisotropic Dirac Cone Reshaping and Ferrimagnetic Spin Polarization in an Organic Conductor, Nat. Commun. 7, 12666 (2016).

[14] V.H. Nguyen and J.-C. Charlier, Klein Tunneling and Electron Optics in Dirac-Weyl Fermion Systems with Tilted Energy Dispersion, Phys. Rev. B 97, 235113 (2018).

[15] C.-K. Chan, N. H. Lindner, G. Refael, and P. A. Lee, Photocurrents in Weyl Semimetals, Phys. Rev. B 95, 041104(R) (2017).

[16] A. A. Soluyanov, D. Gresch, Z. Wang, Q. Wu, M. Troyer, X. Dai, and B. A. Bernevig, Type-II Weyl Semimetals, Nature (London) 527, 495 (2015).

[17] Y. Xu, F. Zhang, and C. Zhang, Structured Weyl Points in Spin-Orbit Coupled Fermionic Superfluids, Phys. Rev. Lett. 115, 265304 (2015).

[18] K. Deng, G. Wan, P. Deng, K. Zhang, S. Ding, E. Wang, M. Yan, H. Huang, H. Zhang, Z. Xu, J. Denlinger, A. Fedorov, H. Yang, W. Duan, H. Yao, Y. Wu, S. Fan, H. Zhang, X. Chen, and S. Zhou, Experimental Observation of Topological Fermi Arcs in Type-II Weyl Semimetal $\mathrm{MoTe}_{2}$, Nat. Phys. 12, 1105 (2016).

[19] L. Huang, T. M. McCormick, M. Ochi, Z. Zhao, M.-T. Suzuki, R. Arita, Y. Wu, D. Mou, H. Cao, J. Yan, N. Trivedi, and A. Kaminski, Spectroscopic Evidence for a Type II Weyl Semimetallic State in MoTe2, Nat. Mater. 15, 1155 (2016).

[20] H.-J. Noh, J. Jeong, E.-J. Cho, K. Kim, B. I. Min, and B.-G. Park, Experimental Realization of Type-II Dirac Fermions in a $\mathrm{PdTe}_{2}$ Superconductor, Phys. Rev. Lett. 119, 016401 (2017).

[21] H. Huang, K.-H. Jin, and F. Liu, Black-Hole Horizon in the Dirac Semimetal $\mathrm{Zn}_{2} \mathrm{In}_{2} \mathrm{~S}_{5}$, Phys. Rev. B 98, 121110(R) (2018).

[22] H. Liu, J.-T. Sun, C. Cheng, F. Liu, and S. Meng, Photoinduced Nonequilibrium Topological States in Strained Black Phosphorus, Phys. Rev. Lett. 120, 237403 (2018).

[23] D. Li, B. Rosenstein, B. Y. Shapiro, and I. Shapiro, Effect of the Type-I to Type-II Weyl Semimetal Topological Transition on Superconductivity, Phys. Rev. B 95, 094513 (2017).

[24] D. Leykam, A. Andreanov, and S. Flach, Artificial Flat Band Systems: From Lattice Models to Experiments, Adv. Phys. X 3, 1473052 (2018).

[25] G. E. Volovik and K. Zhang, Black Hole and Hawking Radiation by Type-II Weyl Fermions, JETP Lett. 104, 645 (2016).

[26] H. Liu, J.-T. Sun, H. Huang, F. Liu, and S. Meng, Fermionic Analogue of Black Hole Radiation with a Super High Hawking Temperature, arXiv:1809.00479.

[27] Y.-G. Chen, X. Luo, F.-Y. Li, B. Chen, and Y. Yu, Quantum Chaos Associated with Emergent Event Horizon in Transition Layer between Type-I and Type-II Weyl Semimetals, arXiv:1903.10886. 
[28] M. Perrot, P. Delplace, and A. Venaille, Topological Transition in Stratified Atmospheres, arXiv:1810.03328.

[29] A. Amo and J. Bloch, Exciton-Polaritons in Lattices: A Non-linear Photonic Simulator, C.R. Phys. 17, 934 (2016).

[30] G. G. Pyrialakos, N. S. Nye, N. V. Kantartzis, and D. N. Christodoulides, Emergence of Type-II Dirac Points in Graphynelike Photonic Lattices, Phys. Rev. Lett. 119, 113901 (2017).

[31] C.-R. Mann, T. J. Sturges, G. Weick, W. L. Barnes, and E. Mariani, Manipulating Type-I and Type-II Dirac Polaritons in Cavity-Embedded Honeycomb Metasurfaces, Nat. Commun. 9, 2194 (2018).

[32] H.-X. Wang, Y. Chen, Z. H. Hang, H.-Y. Kee, and J.-H. Jiang, Type-II Dirac Photons, npj Quantum Mater. 2, 54 (2017).

[33] B. Yang, Q. Guo, B. Tremain, R. Liu, L. E. Barr, Q. Yan, W. Gao, H. Liu, Y. Xiang, J. Chen, C. Fang, A. Hibbins, L. Lu, and S. Zhang, Ideal Weyl Points and Helicoid Surface States in Artificial Photonic Crystal Structures, Science 359, 1013 (2018).

[34] C. Hu, Z. Li, R. Tong, X. Wu, Z. Xia, L. Wang, S. Li, Y. Huang, S. Wang, B. Hou, C. Chan, and W. Wen, Type-II Dirac Photons at Metasurfaces, Phys. Rev. Lett. 121, 024301 (2018).

[35] J. Noh, S. Huang, D. Leykam, Y. D. Chong, K. P. Chen, and M. C. Rechtsman, Experimental Observation of Optical Weyl Points and Fermi Arc-like Surface States, Nat. Phys. 13, 611 (2017).

[36] T. Jacqmin, I. Carusotto, I. Sagnes, M. Abbarchi, D. Solnyshkov, G. Malpuech, E. Galopin, A. Lemaître, J. Bloch, and A. Amo, Direct Observation of Dirac Cones and a Flatband in a Honeycomb Lattice for Polaritons, Phys. Rev. Lett. 112, 116402 (2014).

[37] M. Milićević, T. Ozawa, G. Montambaux, I. Carusotto, E. Galopin, A. Lemaître, L. Le Gratiet, I. Sagnes, J. Bloch, and A. Amo, Orbital Edge States in a Photonic Honeycomb Lattice, Phys. Rev. Lett. 118, 107403 (2017).

[38] V. G. Sala, D. D. Solnyshkov, I. Carusotto, T. Jacqmin, A. Lemaître, H. Terças, A. Nalitov, M. Abbarchi, E. Galopin, I. Sagnes, J. Bloch, G. Malpuech, and A. Amo, Spin-Orbit Coupling for Photons and Polaritons in Microstructures, Phys. Rev. X 5, 011034 (2015).

[39] S. Dufferwiel, F. Li, E. Cancellieri, L. Giriunas, A. A. P. Trichet, D. M. Whittaker, P. M. Walker, F. Fras, E. Clarke, J. M. Smith, M. S. Skolnick, and D. N. Krizhanovskii, Spin Textures of Exciton-Polaritons in a Tunable Microcavity with Large TE-TM Splitting, Phys. Rev. Lett. 115, 246401 (2015).

[40] M. Milićević, T. Ozawa, P. Andreakou, I. Carusotto, T. Jacqmin, E. Galopin, A. Lemaître, L. Le Gratiet, I. Sagnes, J. Bloch, and A. Amo, Edge States in Polariton Honeycomb Lattices, 2D Mater. 2, 034012 (2015).

[41] See Supplemental Material at http://link.aps.org/ supplemental/10.1103/PhysRevX.9.031010 for a description of the experimental setup and additional experimental data.

[42] E. L. Shirley, L. J. Terminello, A. Santoni, and F. J. Himpsel, Brillouin-Zone-Selection Effects in Graphite Photoelectron Angular Distributions, Phys. Rev. B 51, 13614 (1995).
[43] C. Wu and S. Das Sarma, $p_{x, y}$-Orbital Counterpart of Graphene: Cold Atoms in the Honeycomb Optical Lattice, Phys. Rev. B 77, 235107 (2008).

[44] M. Galbiati, L. Ferrier, D. D. Solnyshkov, D. Tanese, E. Wertz, A. Amo, M. Abbarchi, P. Senellart, I. Sagnes, A. Lemaitre, E. Galopin, G. Malpuech, and J. Bloch, Polariton Condensation in Photonic Molecules, Phys. Rev. Lett. 108, 126403 (2012).

[45] G. Montambaux, L.-K. Lim, J.-N. Fuchs, and F. Piéchon, Winding Vector: How to Annihilate Two Dirac Points with the Same Charge, Phys. Rev. Lett. 121, 256402 (2018).

[46] Y. D. Chong, X.-G. Wen, and M. Soljačić, Effective Theory of Quadratic Degeneracies, Phys. Rev. B 77, 235125 (2008).

[47] C. Zeng and V. Elser, Quantum Dimer Calculations on the Spin-1/2 Kagome Heisenberg Antiferromagnet, Phys. Rev. B 51, 8318 (1995).

[48] S. Xia, A. Ramachandran, S. Xia, D. Li, X. Liu, L. Tang, Y. $\mathrm{Hu}, \mathrm{D}$. Song, J. Xu, D. Leykam, S. Flach, and Z. Chen, Unconventional Flatband Line States in Photonic Lieb Lattices, Phys. Rev. Lett. 121, 263902 (2018).

[49] C. Cohen-Tannoudji, J. Grynberg, and G. Dupont-Roc, Atom-Photon Interactions: Basic Process and Applications (Wiley, New York, 1992).

[50] R. de Gail, J.-N. Fuchs, M. O. Goerbig, F. Piéchon, and G. Montambaux, Manipulation of Dirac Points in Graphene-like Crystals, Physica B (Amsterdam) 407, 1948 (2012).

[51] B. Dóra, I. F. Herbut, and R. Moessner, Occurrence of Nematic, Topological, and Berry Phases When a Flat and a Parabolic Band Touch, Phys. Rev. B 90, 045310 (2014).

[52] K. Sun, H. Yao, E. Fradkin, and S. A. Kivelson, Topological Insulators and Nematic Phases from Spontaneous Symmetry Breaking in 2D Fermi Systems with a Quadratic Band Crossing, Phys. Rev. Lett. 103, 046811 (2009).

[53] I. Carusotto and C. Ciuti, Quantum Fluids of Light, Rev. Mod. Phys. 85, 299 (2013).

[54] A. V. Nalitov, D. D. Solnyshkov, and G. Malpuech, Polariton ZTopological Insulator, Phys. Rev. Lett. 114, 116401 (2015).

[55] T. Karzig, C.-E. Bardyn, N. H. Lindner, and G. Refael, Topological Polaritons, Phys. Rev. X 5, 031001 (2015).

[56] P. St.-Jean, V. Goblot, E. Galopin, A. Lemaître, T. Ozawa, L. Le Gratiet, I. Sagnes, J. Bloch, and A. Amo, Lasing in Topological Edge States of a One-Dimensional Lattice, Nat. Photonics 11, 651 (2017).

[57] S. Klembt, T. H. Harder, O. A. Egorov, K. Winkler, R. Ge, M. A. Bandres, M. Emmerling, L. Worschech, T. C. H. Liew, M. Segev, C. Schneider, and S. Höfling, Exciton-Polariton Topological Insulator, Nature (London) 562, 552 (2018).

[58] M. Di Liberto, A. Hemmerich, and C. M. Smith, Topological Varma Superfluid in Optical Lattices, Phys. Rev. Lett. 117, 163001 (2016).

[59] G. Muñoz-Matutano, A. Wood, M. Johnsson, X. Vidal, B. Q. Baragiola, A. Reinhard, A. Lemaître, J. Bloch, A. Amo, G. Nogues, B. Besga, M. Richard, and T. Volz, Emergence of Quantum Correlations from Interacting Fibre-Cavity Polaritons, Nat. Mater. 18, 213 (2019).

[60] A. Delteil, T. Fink, A. Schade, S. Höfling, C. Schneider, and A. Mamolu, Towards Polariton Blockade of Confined Exciton Polaritons, Nat. Mater. 18, 219 (2019). 